\title{
Clinical Utility Gene Card for: Becker muscular dystrophy
}

\author{
David Coote $^{1}$ - Mark R. Davis ${ }^{2}$ - Macarena Cabrera ${ }^{3}$ Merrilee Needham ${ }^{4,5,6,7} \cdot$ Nigel G. Laing $^{1,2} \cdot$ Kristen J. Nowak $^{1,8,9}$
}

Received: 18 January 2017 / Revised: 10 September 2017 / Accepted: 23 November 2017 / Published online: 21 February 2018

(c) European Society of Human Genetics 2018

\section{Disease characteristics}

\subsection{Name of the disease (synonyms)}

Becker muscular dystrophy (BMD).

\subsection{OMIM\# of the disease}

300376

\subsection{Name of the analysed genes or DNA/ chromosome segments}

Dystrophin (DMD)

\subsection{OMIM\# of the gene(s)}

*300377

\subsection{Mutational spectrum}

Variants in the dystrophin/DMD gene can result in Becker or Duchene muscular dystrophy (BMD and DMD, respectively, also known as 'dystrophinopathies') almost solely in male individuals as $D M D$ is located on the $\mathrm{X}$ chromosome. The

Kristen J. Nowak

kristen.nowak@health.wa.gov.au

1 Centre for Medical Research, Faculty of Health and Medical Sciences, The University of Western Australia, and Harry Perkins Institute of Medical Research, QQ Block, QEII Medical Centre, Nedlands, WA 6009, Australia

2 Neurogenetics Laboratory, Department of Diagnostic Genomics, PP Block, QEII Medical Centre, Nedlands, WA 6009, Australia

3 Neurology Department and Instituto de Biomedicina de Sevilla, Hospital Universitario Virgen del Rocío, Seville 41013, Spain

4 Institute for Immunology and Infectious Diseases, Murdoch University, Building 390, Discovery Way, Murdoch Perth, WA 6150, Australia difference in phenotype is usually dependent on whether the variant is in frame, resulting in an internally deleted, shorter, yet partially functional dystrophin protein (BMD), or out-offrame resulting in no dystrophin protein (DMD) [1]. However, other clinical phenotypes may arise from a $D M D$ variant such as isolated quadriceps myopathy [2]; asymptomatic hyperCKemia [3]; myalgia, cramps and rhabdomyolysis [4]; dilated cardiomyopathy [5]; isolated cognitive impairment [6]; and symptomatic female carriers [7].

BMD is less common and less severe than DMD [8]. However, the BMD phenotype is highly variable, with half of affected males presenting by age 10 years with a limbgirdle pattern of skeletal muscle weakness, and often with calf hypertrophy. Other BMD cases are associated with a much milder phenotype and very late onset up to the $7^{\text {th }}$ decade of life, e.g., ref. [9]. Cardiomyopathy is present in $70 \%$ of patients with dystrophinopathies [10], while cognition is usually unaffected. Respiratory involvement usually correlates with the severity of skeletal muscle weakness; therefore, it is more severe in DMD than BMD. Conversely, myocardial involvement does not correlate with skeletal muscle weakness and it should be monitored in every patient. In patients with a childhood onset of skeletal muscle weakness, loss of ambulation may occur in the third or fourth decade of life [8].

5 Murdoch University, 90 South Street, Murdoch, WA 6150, Australia

6 Notre Dame University, 32 Mouat Street, Fremantle, WA 6160, Australia

7 Fiona Stanley Hospital, 11 Robin Warren Drive, Murdoch Perth, WA 6150, Australia

8 School of Biomedical Sciences, Faculty of Health and Medical Sciences, The University of Western Australia, Nedlands, WA 6009, Australia

9 Office of Population Health Genomics, Division of Public and Aboriginal Health, Department of Health, East Perth, WA 6004, Australia 
Regularly updated public $D M D$ variant databases are in the Leiden Open Variation Databases (http://www.dmd.nl/ nmdb2/home.php?select_db = DMD) and ClinVar (https:// www.ncbi.nlm.nih.gov/clinvar/). In-frame deletions (60-70\%) and duplications (5-10\%) account for the majority of BMD cases [11]. Approximately 5-10\% of BMD cases are a result of point variants. The majority of these are small indels and splice site variants, and less commonly missense variants; however, they can also affect dystrophin function $[11,12]$. Intronic variants can also negatively influence splicing and lead to BMD. Detailed understanding of the molecular mechanisms of BMD is important to underpin therapeutic strategies aimed at reducing dystrophinopathy symptoms. In particular, much research emphasis has focussed on the conversion of patients from a DMD-like phenotype to the milder end of the BMD phenotypic spectrum (e.g., a deletion of $46 \%$ of the $D M D$ gene identified in a BMD patient who was still ambulant at 61 years [9] has crucially informed a range of treatment approaches, including use of a 'mini-dystrophin' gene version).

The 'reading frame rule' is not followed in $\sim 10 \%$ of cases [8]. Predicted nonsense variants in exons 23-42 can result in BMD through altering splice definition regions such that the mutated exon is not spliced into the mature mRNA [13, 14]. An out-of-frame deletion of exons 3-7, although usually associated with $\mathrm{DMD}$, can result in BMD because of downstream translational reinitiation (exons numbered as per NG_012232.1 and the Leiden Open Variation Database) $[15,16]$.

\subsection{Analytical methods}

Suspected BMD or DMD patients are first tested for elevated serum CK levels [17]. Mean elevation rates of $\sim 20$ times that of a normal level have been published for BMD patients [17, 18], compared with a 50-100-fold elevation in DMD patients [17]. BMD is characterised by reduced dystrophin expression, which can be visualised using dystrophin antibodies with skeletal muscle biopsy sections. While this remains the gold standard for dystrophinopathy diagnosis (and for differentiating between DMD and BMD), taking skeletal muscle biopsies is invasive and can be avoided in some cases. The DMD phenotype is specific and consistent [19], meaning BMD can often be diagnosed on a differential basis when combined with the identification of a previously characterised BMD-causing variant. This process is facilitated by the presence of an accurate X-linked family tree. When a variant of unknown significance is identified, or a negative genetic test follows clinical suspicion, skeletal muscle biopsy is usually required to facilitate confirmatory testing (e.g., dystrophin immunostaining and transcript analysis).
Although numerous techniques can be used to identify exon copy number variants [12, 20, 21], the multiplex ligation-dependent probe amplification (MLPA) technique [22] and array comparative genomic hybridisation (array $\mathrm{CGH}$ ) are commonly preferred. In the absence of a detected deletion/duplication, the next step is to sequence all $D M D$ exons including intronic flanking regions using a massively parallel sequencing platform. The $D M D$ gene may be sequenced as part of an in-house gene panel, a commercially available sequencing gene panel such as TruSight One (Illumina), or a whole exome. Single-step methods able to detect exon copy variants and exonic point variants are also streamlining this process [23-26]. Point variants affecting splicing (including those deep within intronic regions of $D M D$ ) can affect RNA expression and/or processing. These can be identified via skeletal muscle biopsy followed by (a) sequencing of cDNA derived from skeletal muscle mRNA, which can guide analysis of genomic DNA [8] or (b) RNAseq-based transcriptome analysis [27]. Alternatively, non-invasive skin biopsies can be taken as an RNA source to detect duplications and deletions of two nucleotides or longer, as well as variants affecting splicing in the $D M D$ transcript. This technique further reduces the diagnostic reliance on skeletal muscle biopsies [28].

\subsection{Analytical validation}

Ideally, Sanger sequencing using a fresh dilution of genomic DNA should occur if a putative disease-causing variant is identified through next generation or Sanger sequencing. If possible, the finding of a single-exon duplication or deletion should be confirmed by different methodologies to discount the presence of rare sequence variants or technical artefacts, which might affect probe binding [29]. Because of exceptions to the 'reading-frame rule', variant analysis should not be relied upon solely to offer a differential diagnosis between BMD and DMD. It is prudent to correlate the clinical manifestation with genetic test results, taking into account age of onset, phenotypic presentation and, if available, dystrophin expression from a skeletal muscle biopsy [8].

\subsection{Estimated frequency of the disease}

(Incidence at birth ('birth prevalence') or population prevalence. If known to be variable between ethnic groups, please report):

Approximately 1 in 18,450 live male births [30]. There is a recurrent exon 1 nonsense variant (c. $9 \mathrm{G}>\mathrm{A}$, p.Trp3Ter; NM_004006), most prevalent in European-Americans with one documented case in Italy [31]. Reports are of a mild phenotype with heterogeneous presentation including limb- 
girdle weakness and/or post-exertional myalgia. Ambulation can be lost in late adulthood.

\subsection{Diagnostic setting}

\begin{tabular}{lll} 
& Yes & No \\
A. (Differential) diagnosis & $\bigotimes$ & $\square$ \\
B. Predictive testing & $\bigotimes$ & $\square$ \\
C. Risk assessment in relatives & $\bigotimes$ & $\square$ \\
D. Prenatal & $\square$ & $\square$ \\
\hline
\end{tabular}

Comment:

Diseases to consider when making a differential diagnosis include limb-girdle muscular dystrophies [32], EmeryDreifuss muscular dystrophy and dilated cardiomyopathy [33].

Presymptomatic testing is available for at risk individuals. However, it is difficult to conclusively predict disease progression from a positive predictive/prenatal test result because of exceptions to the 'reading frame rule' and heterogeneity of the BMD phenotype [9, 31, 34].

The same techniques used to provide a molecular diagnosis in the index patient can be used to identify female carrier status and screen other at-risk relatives [29]. Sporadic variants can arise de novo in a female carrier or an index patient $(\sim 33.3 \%$ of all $B M D$ variants) or via germinal mosaicism of the mother [29, 35]. Sons of mosaic mothers are at an elevated risk of developing disease, relative to the level of gonadal mosaicism. There is no elevated risk of developing BMD for family members of de novo carriers/index patients, apart for offspring of these individuals.

\section{Test characteristics}

$\begin{array}{ll}\begin{array}{l}\text { Genotype or A: True-positives } \\ \text { disease }\end{array} & \begin{array}{l}\text { C: False- } \\ \text { negative }\end{array} \\ \text { Present Absent B: False-positives } & \begin{array}{l}\text { D: True- } \\ \text { negative }\end{array}\end{array}$

Test

\begin{tabular}{llll} 
Pos. A & B & Sensitivity:Specificity & A/(A + C $)$ \\
& & & D/(D + B $)$ \\
Neg. C & D & $\begin{array}{l}\text { Pos. predict. value: } \\
\text { Neg. predict. value: }\end{array}$ & A/(A + B $)$ \\
& & D + D $)$ \\
\hline
\end{tabular}

\subsection{Analytical sensitivity}

(proportion of positive tests if the genotype is present) Close to $100 \%$ following exhaustive genetic testing. MLPA has an analytical sensitivity of $\sim 71 \%$ and when combined with Sanger or massively parallel sequencing of the coding regions and splice sites following negative MLPA results, the analytical sensitivity becomes $~ 97 \%$ [29]. Some mutational analyses using single platforms have achieved sensitivities from 92 to $99 \%$ [23, 25, 26]. Array CGH can identify complex rearrangements that go undetected by MLPA [36]. Directed sequencing based on results from sequencing cDNA derived from skeletal muscle mRNA can reveal variants affecting splicing, including those located within deep intronic regions [37].

\subsection{Analytical specificity}

(proportion of negative tests if the genotype is not present) Close to $100 \%$.

\subsection{Clinical sensitivity}

(proportion of positive tests if the disease is present)

The clinical sensitivity can be dependent on variable factors such as age or family history. In such cases a general statement should be given, even if a quantification can only be made case by case.

BMD is more commonly mistaken for related disorders rather than DMD due to its greater phenotypic variability [38] (see section 1.10) and therefore the clinical sensitivity is $85-90 \%$. When BMD is correctly diagnosed clinically, a causative variant is almost always identified if extensive genetic testing is performed, as per section 2.1.

\subsection{Clinical specificity}

(proportion of negative tests if the disease is not present)

The clinical specificity can be dependent on variable factors such as age or family history. In such cases a general statement should be given, even if a quantification can only be made case by case.

Close to $100 \%$.

\subsection{Positive clinical predictive value}

(life time risk to develop the disease if the test is positive).

Below $100 \%$. Some individuals with $D M D$ variants are asymptomatic [39], or present with only slightly elevated serum CK levels and no other phenotype [3]. However, if 
the variant is known to cause BMD in family members, the index patient will almost certainly develop disease. A list of variants resulting in no known pathogenicity can be found at the Leiden Open Variation Database.

\subsection{Negative clinical predictive value}

(Probability not to develop the disease if the test is negative)

Assume an increased risk based on family history for a non-affected person. Allelic and locus heterogeneity may need to be considered.

Index case in that family had been tested:

Close to $100 \%$. An undetected $D M D$ variant may be present that is different to the other previously identified the family. Families have been described that have two different $D M D$-causing variants $[40,41]$.

Index case in that family had not been tested:

Close to $100 \%$.

\section{Clinical utility}

\section{1 (Differential) diagnostics: The tested person is clinically affected}

(To be answered if in 1.9 "A" was marked)

\subsubsection{Can a diagnosis be made other than through a genetic test?}

\begin{tabular}{|c|c|}
\hline Yes $\square$ & \\
\hline Clinically & $\begin{array}{l}\bigotimes \text { Physical examination can be } \\
\text { indicative, however not conclusive }\end{array}$ \\
\hline Imaging & $\square$ \\
\hline Endoscopy & $\square$ \\
\hline Biochemistry & $\begin{array}{l}\otimes \text { Elevated serum CK levels are } \\
\text { suggestive, but require other con- } \\
\text { firmatory diagnostic tests }\end{array}$ \\
\hline Electrophysiology & $\square$ \\
\hline $\begin{array}{l}\text { Other (please } \\
\text { describe): }\end{array}$ & $\begin{array}{l}\text { Histopathology. Dystrophin pro- } \\
\text { tein expression can be detected } \\
\text { using either immunofluorescence } \\
\text { or immunohistochemical staining } \\
\text { of skeletal muscle biopsy sections } \\
\text { or by immunoblot/western blot } \\
\text { analysis of homogenized tissue. } \\
\text { Reduction in expression levels and/ } \\
\text { or a change in dystrophin size can } \\
\text { be indicative of BMD }\end{array}$ \\
\hline
\end{tabular}

\subsubsection{Describe the burden of alternative diagnostic methods to the patient}

Clinical and biochemical assessment can usually offer a preliminary diagnosis, although genetic testing is required to confirm the diagnosis, determine the causative variant and thus the disease mechanism in most cases. Failure to identify a causative $D M D$ variant complicates predictive testing in relatives and genetic counselling for potential female carriers. In addition, genetic testing streamlines the diagnostic process considerably [42] and, in some cases, avoids the need for a skeletal muscle biopsy, which can be distressing for some patients.

\subsubsection{How is the cost effectiveness of alternative diagnostic methods to be judged?}

Additional diagnostic cost most likely results from utilising alternative diagnostic methodologies, as a molecular diagnosis is the desired outcome. However, some economic benefit may arise if alternative diagnostic methods can direct molecular analysis, such as indicating whether any dystrophin protein is expressed, and if so, how the protein may be perturbed in size. In such instances a molecular diagnosis may arise more quickly than without the added information.

\subsubsection{Will disease management be influenced by the result of a genetic test?}

No.

Yes. $\otimes$

Therapy (please describe)
Currently there are no disease modifying treatments for BMD. Corticosteroids which are recommended for DMD patients are generally avoided due to their adverse side effects, although they can prolong ambulation in some severe cases [43]. Most geneticbased therapies being trialled experimentally and clinically aim to convert a DMD phenotype to a BMD phenotype, so will probably only be applicable for patients with a more severe version of BMD [8]. Pharmacological approaches targeting secondary pathology (e.g. calcium dysregulation) downstream of the deficiency of normal 
Table (continued)

$\begin{array}{ll}\text { dystrophin in DMD may also } \\ \text { prove to be helpful for BMD [44]. } \\ \text { describe) } & \text { BMD is associated with a highly } \\ \text { heterogeneous phenotype and as a } \\ \text { result prognosis can rarely be } \\ \text { accurately predicted from genetic } \\ \text { tests alone. Even if a non-private } \\ \text { variant is found, large variation in } \\ \text { phenotype within families has } \\ \text { been reported. However, variants } \\ \text { in domain I, responsible for actin } \\ \text { binding, usually result in severe } \\ \text { BMD, and also intermediate mus- } \\ \text { cular dystrophy (characterised by } \\ \text { loss of ambulation between the age } \\ \text { of } 12 \text { and 15 years) [13]. Domain } \\ \text { II (the rod domain) is not essential } \\ \text { for protein function and thus } \\ \text { variants in this region are often } \\ \text { associated with a mild phenotype } \\ \text { and occasionally asymptomatic } \\ \text { individuals [39, 45]. } \\ \text { Confirming the diagnosis of a } \\ \text { dystrophinopathy should prompt } \\ \text { cardiac screening. Variants in } \\ \text { DMD can cause myocardial com- } \\ \text { plications, including in asympto- } \\ \text { matic individuals and carrier } \\ \text { females [19, } 46] . \text { BMD is managed } \\ \text { in a similar fashion to DMD } \\ \text { (reviewed by Bushby et al.) } \\ \text { [19, 47]. In brief, symptoms can be } \\ \text { alleviated through physiotherapy, } \\ \text { occupational therapy and mobility } \\ \text { and ventilation aids. Each is } \\ \text { recommended on a case-to-case } \\ \text { basis depending on disease sever- } \\ \text { ity and progression, not on genetic } \\ \text { diagnosis. }\end{array}$

3.2 Predictive Setting: the tested person is clinically unaffected but carries an increased risk based on family history

(To be answered if in 1.9 ' $\mathrm{B}$ ' was marked)

\subsubsection{Will the result of a genetic test influence lifestyle and prevention?}

If the test result is positive (please describe)
There is little evidence to suggest any benefit of presymptomatic therapy for BMD. However, an early diagnosis can have a significant effect on both the lifestyle of the patient and their family. Ambulation can be lost in early- or mid-adulthood and thus a positive result may allow a patient to minimise their reliance on independent mobility. This could include purchase of a single story home, and pursuing a sedentary career as apposed to one that requires physical work. A confirmatory genetic diagnosis is also likely to result in cardiac monitoring and management of any cardiac complications. For the family, early diagnosis in a child can prompt carrier testing in the mother and other female relatives, and in turn provide various genetic counselling options such as prenatal and preimplantation diagnosis.

If the test result is negative (please describe)

A negative test result in an asymptomatic at-risk male will provide obvious relief for the individual and his family, and influence their lifestyle choices in the opposite manner as outlined above. A negative test result for a potential female carrier provides reassurance in regards to future family planning.

\subsubsection{Which options in view of lifestyle and prevention does} a person at-risk have if no genetic test has been done (please describe)?

Indicative diagnostic tests are available (see 3.1.1). The same lifestyle and prevention options exist as for a person with a positive test result.

\subsection{Genetic risk assessment in family members of a diseased person}

(To be answered if in 1.9 ' $\mathrm{C}$ ' was marked)

\subsubsection{Does the result of a genetic test resolve the genetic situation in that family?}

Yes, if a disease-causing variant is found in the diseased person, then a genetic test for family members can determine their risk above that of the general population.

\subsubsection{Can a genetic test in the index patient save genetic or other tests in family members?}

If, due to family history, a mother is known to carry a variant that has not yet been characterised, a positive genetic test in an affected child can confirm the variant identity in the mother. If the mother of an affected child is of unknown carrier status, it is recommended she be tested, as the result affects future family planning. Testing of symptomatic brothers or close male (and female in rare 
situations) relatives of an index patient is still warranted, although the process is refined if the disease-causing variant has already been characterised in the index patient. If the variant is a deletion or a duplication, an exon copy number test can be used (see section 1.6). For other variants, sequence analysis focusing on the area of the gene containing the variant is standard.

\subsubsection{Does a positive genetic test result in the index patient enable a predictive test in a family member?}

Yes. The carrier status for the mother of an affected child will determine whether the disease-causing variant in the index patient has been inherited or occurred de novo. If inherited, cascade testing of females at risk of being carriers and X-linked male relatives (including those older than the index patient) is also warranted. It is possible that the disease-causing variant in the index case could result in a vast spectrum of disease severity, including the age of onset [9]. A positive result in the index patient's relatives would nevertheless allow genetic counselling options to be provided.

\subsection{Prenatal diagnosis}

(To be answered if in 1.9 'D' was marked)

\subsubsection{Does a positive genetic test result in the index patient enable a prenatal diagnosis?}

Yes. When a mother is a known or suspected carrier then prenatal testing is usually considered. In this case, a differential diagnosis between BMD and DMD is made easier by the family history and whether the $D M D$ variant perturbs the reading frame or not. However, phenotypic variability and exceptions to the reading frame rule make it difficult to make conclusive prognostic predictions from a prenatal genetic test alone.

\section{If applicable, further consequences of testing}

Please assume that the result of a genetic test has no immediate medical consequences. Is there is any evidence that a genetic test is nevertheless useful for the patient or his/her relatives? (Please describe)

A positive genetic test will have practical and psychosocial consequences on patients and families. In the case of a presymptomatic test, the convoluted clinical diagnostic process can be avoided, eliminating the anxiety associated with misunderstanding early symptoms [48]. It can also predict the probable prognosis of some patients, allowing time for them and their families to prepare emotionally and practically for disease onset. Finally, a conclusive genetic test usually prompts cascade testing of at-risk males and potential female carriers, providing information that forms the basis of genetic counselling and allows families to consider preimplantation or prenatal testing.

Acknowledgements This work was supported by EuroGentest2 (Unit 2: 'Genetic testing as part of health care'), a Coordination Action under FP7 (Grant Agreement Number 261469) and the European Society of Human Genetics. K.J.N. was funded by an Australian Research Council Future Fellowship FT100100734, N.G.L. by a National Health and Medical Research Council Principal Research Fellowship APP1002147 and M.C. by Instituto de Salud Carlos III (JR15/00042).

\section{Compliance with ethical standards}

Conflict of interest The authors declare that they have no conflict of interest.

\section{References}

1. Hoffman EP, Brown RH Jr, Kunkel LM. Dystrophin: the protein product of the Duchenne muscular dystrophy locus. Cell. 1987;51:919-28.

2. Sunohara N, Arahata K, Hoffman EP, et al. Quadriceps myopathy: forme fruste of Becker muscular dystrophy. Ann Neurol. 1990;28:634-9.

3. Melis MA, Cau M, Muntoni F, et al. Elevation of serum creatine kinase as the only manifestation of an intragenic deletion of the dystrophin gene in three unrelated families. Eur J Paediatr Neurol. 1998;2:255-61.

4. Veerapandiyan A, Shashi V, Jiang YH, Gallentine WB, Schoch K, Smith EC. Pseudometabolic presentation of dystrophinopathy due to a missense mutation. Muscle Nerve. 2010;42:975-9.

5. Nakamura A. X-linked dilated cardiomyopathy: a cardiospecific phenotype of dystrophinopathy. Pharmaceuticals. 2015;8: 303-20.

6. North KN, Miller G, Iannaccone ST, et al. Cognitive dysfunction as the major presenting feature of Becker's muscular dystrophy. Neurology. 1996;46:461-5.

7. Giliberto F, Radic CP, Luce L, Ferreiro V, de Brasi C, Szijan I. Symptomatic female carriers of Duchenne muscular dystrophy (DMD): genetic and clinical characterization. J Neurol Sci. 2014;336:36-41.

8. Wein N, Alfano L, Flanigan KM. Genetics and emerging treatments for Duchenne and Becker muscular dystrophy. Pediatr Clin North Am. 2015;62:723-42.

9. England SB, Nicholson LV, Johnson MA, et al. Very mild muscular dystrophy associated with the deletion of $46 \%$ of dystrophin. Nature. 1990;343:180-2.

10. Kaspar RW, Allen HD, Ray WC, et al. Analysis of dystrophin deletion mutations predicts age of cardiomyopathy onset in becker muscular dystrophy. Circ Cardiovasc Genet. 2009;2:544-51.

11. Takeshima Y, Yagi M, Okizuka Y, et al. Mutation spectrum of the dystrophin gene in 442 Duchenne/Becker muscular dystrophy cases from one Japanese referral center. J Hum Genet. 2010;55:379-88.

12. Flanigan KM, von Niederhausern A, Dunn DM, Alder J, Mendell JR, Weiss RB. Rapid direct sequence analysis of the dystrophin gene. Am J Hum Genet. 2003;72:931-9. 
13. Flanigan KM, Dunn DM, von Niederhausern A, et al. Nonsense mutation-associated Becker muscular dystrophy: interplay between exon definition and splicing regulatory elements within the DMD gene. Hum Mutat. 2011;32:299-308.

14. Disset A, Bourgeois CF, Benmalek N, Claustres M, Stevenin J, Tuffery-Giraud S. An exon skipping-associated nonsense mutation in the dystrophin gene uncovers a complex interplay between multiple antagonistic splicing elements. Hum Mol Genet. 2006;15:999-1013.

15. Gangopadhyay SB, Sherratt TG, Heckmatt JZ, et al. Dystrophin in frameshift deletion patients with becker muscular dystrophy. Am J Hum Genet. 1992;51:562-70.

16. Winnard AV, Mendell JR, Prior TW, Florence J, Burghes AHM. Frameshift deletions of exons 3-7 and revertant fibers in Duchenne muscular dystrophy: mechanisms of dystrophin production. Am J Hum Genet. 1995;56:158-66.

17. Zatz M, Rapaport D, Vainzof M, et al. Serum creatine-kinase (CK) and pyruvate-kinase (PK) activities in Duchenne (DMD) as compared with Becker (BMD) muscular dystrophy. J Neurol Sci. 1991;102:190-6.

18. Burch PM, Pogoryelova O, Goldstein R, et al. Muscle-derived proteins as serum biomarkers for monitoring disease progression in three forms of muscular dystrophy. J Neuromuscul Dis. 2015;2:241-55.

19. Bushby K, Finkel R, Birnkrant DJ, et al. Diagnosis and management of Duchenne muscular dystrophy, part 1: diagnosis, and pharmacological and psychosocial management. Lancet Neurol. 2010;9:77-93.

20. Prior TW, Bridgeman SJ. Experience and strategy for the molecular testing of Duchenne muscular dystrophy. J Mol Diagn. 2005;7:317-26.

21. del Gaudio D, Yang Y, Boggs BA, et al. Molecular diagnosis of Duchenne/Becker muscular dystrophy: enhanced detection of dystrophin gene rearrangements by oligonucleotide arraycomparative genomic hybridization. Hum Mutat. 2008;29:1100-7.

22. Janssen B, Hartmann C, Scholz V, Jauch A, Zschocke J. MLPA analysis for the detection of deletions, duplications and complex rearrangements in the dystrophin gene: potential and pitfalls. Neurogenetics. 2005;6:29-35.

23. Lim BC, Lee S, Shin JY, et al. Genetic diagnosis of Duchenne and Becker muscular dystrophy using next-generation sequencing technology: comprehensive mutational search in a single platform. J Med Genet. 2011;48:731-6.

24. Wang Y, Yang Y, Liu J, et al. Whole dystrophin gene analysis by next-generation sequencing: a comprehensive genetic diagnosis of Duchenne and Becker muscular dystrophy. Mol Genet Genome. 2014;289:1013-21.

25. Wei X, Dai Y, Yu P, et al. Targeted next-generation sequencing as a comprehensive test for patients with and female carriers of DMD/BMD: a multi-population diagnostic study. Eur J Hum Genet. 2014;22:110-8.

26. Okubo M, Minami N, Goto $\mathrm{K}$, et al. Genetic diagnosis of Duchenne/Becker muscular dystrophy using next-generation sequencing: validation analysis of DMD mutations. J Hum Genet. 2016;61:483-9.

27. Gonorazky H, Liang M, Cummings B, et al. RNAseq analysis for the diagnosis of muscular dystrophy. Ann Clin Transl Neurol. 2016;3:55-60.

28. Bovolenta M, Scotton C, Falzarano MS, Gualandi F, Ferlini A. Rapid, comprehensive analysis of the dystrophin transcript by a custom micro-fluidic exome array. Hum Mutat. 2012;33:572-81.
29. Grimm T, Kress W, Meng G, Müller CR. Risk assessment and genetic counseling in families with Duchenne muscular dystrophy. Acta Myol. 2012;31:179-83.

30. Bushby KMD, Thambyayah M, Gardner-Medwin D. Prevalence and incidence of Becker muscular dystrophy. Lancet. 1991;337:1022-4.

31. Flanigan KM, Dunn DM, von Niederhausern A, et al. DMD Trp3X nonsense mutation associated with a founder effect in North American families with mild Becker muscular dystrophy. Neuromuscul Disord. 2009;19:743-8.

32. Schwartz M, Hertz JM, Sveen ML, Vissing J. LGMD2I presenting with a characteristic Duchenne or Becker muscular dystrophy phenotype. Neurology. 2005;64:1635-7.

33. Darras BT, Miller DT, Urion DK. Dystrophinopathies. In: Pagon RA, Adam MP, Ardinger HH et al., editors. Gene Reviews. Washington, Seattle; 2000. https://www.ncbi.nlm.nih.gov/ pubmed/20301298

34. Bushby KMD, Gardner-Medwin D. The clinical, genetic and dystrophin characteristics of Becker muscular dystrophy. J Neurol. 1993;240:98-104.

35. Edwards JH. The population genetics of Duchenne: natural and artificial selection in Duchenne muscular dystrophy. J Med Genet. 1986;23:521-30.

36. Dd Gaudio, Yang Y, Boggs BA, et al. Molecular diagnosis of Duchenne/Becker muscular dystrophy: enhanced detection of dystrophin gene rearrangements by oligonucleotide arraycomparative genomic hybridization. Hum Mutat. 2008;29:1100-7.

37. Mah JK, Selby K, Campbell C, et al. A population-based study of dystrophin mutations in Canada. Can $J$ Neurol Sci. 2011;38:465-74.

38. Kesari A, Pirra LN, Bremadesam L, et al. Integrated DNA, cDNA, and protein studies in Becker muscular dystrophy show high exception to the reading frame rule. Hum Mutat. 2008;29:728-37.

39. Ferreiro V, Giliberto F, Muñiz GMN, et al. Asymptomatic Becker muscular dystrophy in a family with a multiexon deletion. Muscle Nerve. 2009;39:239-43.

40. Wilton SD, Johnsen RD, Pedretti JR, Laing NG. Two distinct mutations in a single dystrophin gene: identification of an altered splice-site as the primary Becker muscular dystrophy mutation. Am J Med Genet. 1993;46:563-9.

41. Morandi L, Mora M, Tedeschi S, et al. DMD and BMD in the same family due to distinct mutations. Am J Med Genet. 1995;59:501-5.

42. Ross LF. Screening for conditions that do not meet the Wilson and Jungner criteria: the case of Duchenne muscular dystrophy. Am J Med Genet A. 2006;140:914-22.

43. Johnsen SD. Prednisone therapy in Becker's muscular dystrophy. J Child Neurol. 2001;16:870-1.

44. Guiraud S and Davies KE. Pharmacological advances for treatment in Duchenne muscular dystrophy. Curr Opin Pharmacol. 2017;34:36-48.

45. Ramelli GP, Joncourt F, Luetschg J, Weis J, Tolnay M, Burgunder JM. Becker muscular dystrophy with marked divergence between clinical and molecular genetic findings: case series. Swiss Med Wkly. 2006;136:189-93.

46. Finsterer J, Stöllberger C. Cardiac involvement in Becker muscular dystrophy. Can J Cardiol. 2008;24:786-92.

47. Bushby K, Finkel R, Birnkrant DJ, et al. Diagnosis and management of Duchenne muscular dystrophy, part 2: implementation of multidisciplinary care. Lancet Neurol. 2010;9:177-89.

48. Parsons E, Clarke A, Hood K, Lycett E, Bradley D. Newborn screening for Duchenne muscular dystrophy: a psychosocial study. Fetal Neonatal Ed. 2002;86:91-95. 